\title{
Charge gravastars in $f(T)$ modified gravity
}

\author{
Ujjal Debnath $^{\mathrm{a}}$ \\ Department of Mathematics, Indian Institute of Engineering Science and Technology, Shibpur, Howrah 711 103, India
}

Received: 14 March 2019 / Accepted: 2 June 2019 / Published online: 13 June 2019

(C) The Author(s) 2019

\begin{abstract}
In the present work, we discus the four dimensional spherically symmetric stellar system in the framework of modified $f(T)$ gravity theory with electromagnetic field. The field equations are written for two cases, either $T^{\prime}=0$ or $f_{T T}=0$. Next we discuss the charged gravastar model which has three regions: an interior region, a shell region and an exterior region. In the interior region, we find the solutions of all physical quantities like density, pressure, electromagnetic field and the metric coefficients for both cases. For $T^{\prime}=0$, a gravastar cannot form, but it forms only for the case $f_{T T}=0$. In the exterior region, we obtain the exterior solution for the vacuum model. In the shell region, we assume that the interior and exterior regions join together at a certain place, so the intermediate region must be thin shell with the approximation $h\left(\equiv e^{-b}\right) \ll 1$. In this approximation, we find the analytical solutions. The proper length of the thin shell, the entropy and the energy content inside the thin shell are found and they are directly proportional to the proper thickness of the shell $\epsilon$ under the approximation $(\epsilon \ll 1)$. According to the Darmois-Israel formalism, we study the matching between the surfaces of interior and exterior regions of the gravastar. The energy density, pressure, equation of state parameter for the surface and mass of the thin shell are obtained.
\end{abstract}

\section{Introduction}

In 2001, Mazur and Mottola [1,2] have found a solution for the gravitationally collapsing neutral system in the concept of Bose-Einstein condensation to gravitational systems. These describe as super compact, spherically symmetric and singularity-free objects that can be considered to be virtually as compact as the black holes. These gravitationally dark cold vacuum compact star is known as gravastar ("gravitational vacuum condensate stars"). The gravastar is a substi-

\footnotetext{
a e-mail: ujjaldebnath @ gmail.com
}

tute of a black hole, i.e., there exist compact stars minus event horizons; this compresses the matter within the gravitational radius $R=\frac{2 \mathrm{GM}}{c^{2}}$, that is, very close to the Schwarzschild radius. In this sense, the existence of quantum vacuum fluctuations are predicted near the event horizon. The gravastar consists of (i) an interior de Sitter condensate phase and (ii) an exterior Schwarzschild geometry. The gravastar consists of five layers with two infinitely thin shells, apparently, on the regions $r=r_{1}$ and $r=r_{2}$ where $r_{1}$ and $r_{2}$ are the inner and the outer radii $\left(r_{1}<r_{2}\right)$. Also other important regions are (i) the interior region: $0 \leq r<r_{1}$ with equation of state (EOS) $p=-\rho$, which is defined by the de Sitter spacetime, (ii) the shell region: $r_{1}<r<r_{2}$ with EOS $p=\rho$, i.e., an intermediary thin layer made of an ultra-stiff perfect fluid, (iii) an exterior region: $r_{2}<r$ with $\mathrm{EOS} p=\rho=0$ (vacuum), which is described by the Schwarzschild solution. Thus the interior region of the gravastar is surrounded by a thin shell of ultra-relativistic matter, whereas the exterior region is completely vacuum. Also, if we replace two infinitely thin stiff shells and the intermediary region with just one infinitely thin shell core [3], then the five layer models can be simplified to three layer models.

There is a lot of work on the gravastar models available in the literature in the framework of Einstein's general relativity. DeBenedictis et al. [4] have found gravastar solutions with continuous pressures and equation of state. Cattoen et al. [5] have taken an anisotropic pressure in the formation of gravastar. Born-Infeld phantom gravastars have been discussed by Bilic et al. [6]. A gravastar model in higher dimensional spacetime has been discussed in Refs. [7-10]. The gravastar in the frame of conformal motion has also been analyzed by some authors $[8,11,12]$. Physical features of charged gravastar have been investigated [8,9,11,13-17]. The stable gravastar is discussed by several authors [18-22].

One important family of modifications of the EinsteinHilbert action is the $f(R)$ theories of gravity [23-26]. In such theories, one uses a function of the curvature scalar as the Lagrangian density. Along similar lines, one can also 
modify the theory to find a teleparallel equivalent of general relativity where the Lagrangian density is equivalent to the torsion scalar $T$ and the field equations of teleparallel gravity [27-31] are identical to the Einsteins field equations in any background metric. Subsequently teleparallel gravity has been modified by having a Lagrangian density equivalent to a function of torsion scalar i.e., $f(T)$ gravity [32]. In theoretical astrophysics, an $f(T)$ version of three dimensions of the BTZ black hole solutions has been calculated as $f(T)$ gravity theory was supported for examining the effects of $f(T)$ gravity models [33]. Recently, static solutions in the spherically symmetric case with a charged source in $f(T)$ gravity have been found [34].

Deliduman et al. [35] have investigated the structure of neutron stars in modified $f(T)$ gravity models. Boehmer et al. [36] have analyzed the existence of relativistic stars in $f(T)$ modified gravity and explicitly constructed several classes of a static perfect fluid solution. Anisotropic strange quintessence stars in $f(T)$ gravity models have been studied in Refs. [37,38]. Compact stars of emending class one in $f(T)$ gravity have been studied in [39]. Strong magnetic field effects on neutron stars within $f(T)$ theory of gravity have been discussed in [40]. Tolman-Oppenheimer-Volkoff equations and their implications for the structures of relativistic stars in $f(T)$ gravity have been found [41]. Abbas and collaborators [42-45] have discussed the anisotropic compact star models in GR, $f(R), f(G)$ and $f(T)$ theories in a diagonal tetrad case with Krori and Barua (KB) metric [46]. The gravastar model in $f(G, \mathcal{T})$ gravity has been discussed by Shamir et al. [47]. Das et al. [48] have studied the gravastar model in modified $f(R, \mathcal{T})$ gravity.

The main motivation of the work is to study the gravastar in the framework of $f(T)$ gravity with the electromagnetic source and examine the nature of the physical parameters in the thin shell region. The organization of the work is as follows: In Sect. 2, we present some brief reviews of $f(T)$ gravity with electromagnetic field. In Sect. 3, we write the Einstein-Maxwell field equations for a spherically symmetric stellar metric in the framework of $f(T)$ gravity theory. In Sect. 4, we address the three regions of the gravastar model and find the metric coefficients and other physical quantities. In Sect. 5, the physical features of gravastar parameters are analyzed. The junction conditions between interior and exterior regions are studied in Sect. 6. Some physical analysis and conclusions of the work are presented in Sect. 7.

\section{$2 f(T)$ Modified gravity and electromagnetic field}

We consider the torsion and the con-torsion tensor as follows [37]:

$$
\begin{aligned}
T_{\mu \nu}^{\alpha} & =\Gamma_{v \mu}^{\alpha}-\Gamma_{\mu \nu}^{\alpha}=e_{i}^{\alpha}\left(\partial_{\mu} e_{\nu}^{i}-\partial_{\nu} e_{\mu}^{i}\right), \\
K_{\alpha}^{\mu \nu} & =-\frac{1}{2}\left(T_{\alpha}^{\mu \nu}-T_{\alpha}^{v \mu}-T_{\alpha}^{\mu \nu}\right),
\end{aligned}
$$

and the components of the tensor $S_{\alpha}^{\mu \nu}$ are defined as

$S_{\alpha}^{\mu \nu}=\frac{1}{2}\left(K_{\alpha}^{\mu \nu}+\delta_{\alpha}^{\mu} T_{\beta}^{\beta \nu}-\delta_{\alpha}^{\nu} T_{\beta}^{\beta \mu}\right)$

So one can write the torsion scalar as in the following form:

$T=T_{\mu \nu}^{\alpha} S_{\alpha}^{\mu \nu}$

the importance of this will become clear in a moment. Now, define the modified teleparallel action by replacing $T$ with a function $f(T)[49,50]$ as follows:

$S=\int d^{4} x \sqrt{-g}\left[\frac{1}{16 \pi} f(T)+\mathcal{L}_{\text {matter }}\right]$

where we choose $G=c=1$ and $\mathcal{L}_{\text {matter }}$ is the matter Lagrangian.

The ordinary matter is an anisotropic fluid so that the energy-momentum tensor is given by

$T_{\mu \nu}^{\text {matter }}=(\rho+p) u_{\mu} u_{\nu}+p g_{\mu \nu}$

where $u^{\mu}$ is the fluid four-velocity satisfying $u_{\mu} u^{\nu}=-1, \rho$ is the energy density of fluid and $p$ is the fluid pressure. Further, the energy-momentum tensor for the electromagnetic field is given by

$T_{\mu \nu}^{E M}=-\frac{1}{4 \pi}\left(g^{\delta \omega} F_{\mu \delta} F_{\omega \nu}-\frac{1}{4} g_{\mu \nu} F_{\delta \omega} F^{\delta \omega}\right)$

where $F_{\mu \nu}$ is the Maxwell field tensor defined as

$F_{\mu \nu}=\Phi_{\nu, \mu}-\Phi_{\mu, \nu}$

and $\Phi_{\mu}$ is the four-potential. The corresponding Maxwell electromagnetic field equations are

$\left(\sqrt{-g} F^{\mu \nu}\right),{ }_{\nu}=4 \pi J^{\mu} \sqrt{-g}, F_{[\mu \nu, \delta]}=0$

where $J^{\mu}$ is the current four-vector satisfying $J^{\mu}=\sigma u^{\mu}$, the parameter $\sigma$ is the charge density.

\section{Einstein-Maxwell field equations}

We consider the spherically symmetric metric describing the interior spacetime as [46]

$$
d s^{2}=-e^{a(r)} \mathrm{d} t^{2}+e^{b(r)} \mathrm{d} r^{2}+r^{2}\left(\mathrm{~d} \theta^{2}+\sin ^{2} \theta \mathrm{d} \phi^{2}\right)
$$


where $a(r)$ and $b(r)$ are functions of $r$. For this metric, we get the torsion scalar $T$ and its derivative $T^{\prime}$ in the following forms [38]:

$$
\begin{aligned}
T(r) & =\frac{2 \mathrm{e}^{-b}}{r}\left(a^{\prime}+\frac{1}{r}\right), \\
T^{\prime}(r) & =\frac{2 \mathrm{e}^{-b}}{r}\left[a^{\prime \prime}-\frac{1}{r^{2}}-\left(a^{\prime}+\frac{1}{r}\right)\left(b^{\prime}+\frac{1}{r}\right)\right],
\end{aligned}
$$

where the prime' denotes the derivative with respect to the radial coordinate $r$.

For the charged fluid source with density $\rho(r)$, pressure $p(r)$ and electromagnetic field $E(r)$, the Einstein-Maxwell (EM) equations reduce to the form $(G=c=1)[36,37]$

$$
\begin{aligned}
& -2 \frac{\mathrm{e}^{-b}}{r} T^{\prime} f_{T T}+\frac{f}{2}-\left(T-\frac{1}{r^{2}}-\frac{\mathrm{e}^{-b}}{r}\left(a^{\prime}+b^{\prime}\right)\right) f_{T} \\
& =8 \pi \rho+E^{2}, \\
& \left(T-\frac{1}{r^{2}}\right) f_{T}-\frac{f}{2}=8 \pi p-E^{2}, \\
& \mathrm{e}^{-b}\left(\frac{a^{\prime}}{2}+\frac{1}{r}\right) T^{\prime} f_{T T}+\left[\frac{T}{2}+\mathrm{e}^{-b}\left\{\frac{a^{\prime \prime}}{2}+\left(\frac{a^{\prime}}{4}+\frac{1}{2 r}\right)\right.\right. \\
& \left.\left.\quad \times\left(a^{\prime}-b^{\prime}\right)\right\}\right] f_{T}-\frac{f}{2}=8 \pi p+E^{2}, \\
& \frac{\mathrm{e}^{-\frac{b}{2}} \cot \theta}{2 r^{2}} T^{\prime} f_{T T}=0 .
\end{aligned}
$$

Adding Eqs. (13) and (14), we obtain

$$
\rho+p=\frac{\mathrm{e}^{-b}}{8 \pi r}\left(a^{\prime}+b^{\prime}\right) f_{T}
$$

Taking the derivative of Eq. (14) and using Eqs. (11) and (17), we obtain the energy conservation equation [9]

$p^{\prime}+\frac{1}{2}(\rho+p) a^{\prime}=\frac{1}{8 \pi r^{4}}\left(r^{4} E^{2}\right)^{\prime}$,

and the electric field $E$ is as follows:

$$
E(r)=\frac{1}{r^{2}} \int_{0}^{r} 4 \pi r^{2} \sigma(r) \mathrm{e}^{\frac{b}{2}} \mathrm{~d} r=\frac{q(r)}{r^{2}}
$$

where $q(r)$ is the total charge within a sphere of radius $r$. The term $\sigma \mathrm{e}^{\frac{b}{2}}$ inside the above integral is known as the volume charge density. The gravitational mass can be written as [9]

$M(r)=\int_{0}^{r} 4 \pi r^{2}\left(\rho+\frac{E^{2}}{8 \pi}\right) \mathrm{d} r$.

From Eq. (16), we get either $T^{\prime}=0$ or $f_{T T}=0$.

Case I: $T^{\prime}=0 \Rightarrow T=$ constant (for all $r$ ) $=T_{0}$, i.e., the torsion scalar $T$ is independent of $r$ and hence
$f(T), f_{T}, f_{T T}, \ldots$ are always constant. Assume $f\left(T_{0}\right)=$ $f_{0}$ and $f_{T}\left(T_{0}\right)=f_{1}$. So Eqs. (13), (14) and (15) reduce to

$$
\begin{aligned}
& \frac{f_{0}}{2}-\left(T_{0}-\frac{1}{r^{2}}-\frac{\mathrm{e}^{-b}}{r}\left(a^{\prime}+b^{\prime}\right)\right) f_{1}=8 \pi \rho+E^{2} \\
& \left(T_{0}-\frac{1}{r^{2}}\right) f_{1}-\frac{f_{0}}{2}=8 \pi p-E^{2} \\
& {\left[\frac{T_{0}}{2}+\mathrm{e}^{-b}\left\{\frac{a^{\prime \prime}}{2}+\left(\frac{a^{\prime}}{4}+\frac{1}{2 r}\right)\left(a^{\prime}-b^{\prime}\right)\right\}\right] f_{1}-\frac{f_{0}}{2}} \\
& \quad=8 \pi p+E^{2}
\end{aligned}
$$

Case II: $f_{T T}=0 \Rightarrow f(T)=\alpha T+\beta$, where $\alpha$ and $\beta$ are constants. So $f(T)$ is a linear function of the torsion scalar $T$. If $\alpha=1$ and $\beta=0$, we get back the usual $T$ model and in this case the field equations will be the same as te usual Ricci scalar $R$ model. Equations (13), (14) and (15) reduce to

$$
\begin{aligned}
& \frac{\alpha \mathrm{e}^{-b}}{r}\left(b^{\prime}-\frac{1}{r}\right)+\frac{\alpha}{r^{2}}+\frac{\beta}{2}=8 \pi \rho+E^{2} \\
& \frac{\alpha \mathrm{e}^{-b}}{r}\left(a^{\prime}+\frac{1}{r}\right)-\frac{\alpha}{r^{2}}-\frac{\beta}{2}=8 \pi p-E^{2} \\
& \alpha \mathrm{e}^{-b}\left[\frac{a^{\prime \prime}}{2}+\left(\frac{a^{\prime}}{4}+\frac{1}{2 r}\right)\left(a^{\prime}-b^{\prime}\right)\right]-\frac{\beta}{2}=8 \pi p+E^{2}
\end{aligned}
$$

\section{Geometry of gravastars}

In this section we will derive the solutions of the field equations for a gravastar and discuss its physical and geometrical interpretations. For the geometrical regions of the gravastar, it is supposed to be extremely thin having a finite width within the regions $D=r_{1}<r<r_{2}=D+\epsilon$ where $r_{1}$ and $r_{2}$ are the radii of the interior and exterior regions of the gravastar and $\epsilon$ is a positive small quantity. In these regions, the equation of state (EOS) parameter is structured as follows: (i) interior region $R_{1}: 0 \leq r<r_{1}$ with EOS $p=-\rho$, (ii) shell region $R_{2}: r_{1}<r<r_{2}$ with EOS $p=\rho$, (iii) exterior region $R_{3}$ : $r_{2}<r$ with $\operatorname{EOS} p=\rho=0$.

\subsection{Interior region}

The equation of state for interior region $R_{1}\left(0 \leq r<r_{1}=D\right)$ of the gravastar is $p=-\rho$. From Eq. (17), we obtain

$\mathrm{e}^{b(r)}=k \mathrm{e}^{-a(r)}$

where $k$ is a constant $>0$.

Case I: $T^{\prime}=0$. 
For $T^{\prime}=0$, we obtain the solutions

$\mathrm{e}^{a(r)}=\frac{k T_{0} r^{3}+C}{6 r}$

and

$\mathrm{e}^{b(r)}=\frac{6 k r}{k T_{0} r^{3}+C}$

where $C$ is constant. So in this case, the metric becomes

$$
\begin{aligned}
\mathrm{d} s^{2}= & -\frac{k T_{0} r^{3}+C}{6 r} \mathrm{~d} t^{2}+\frac{6 k r}{k T_{0} r^{3}+C} \mathrm{~d} r^{2} \\
& +r^{2}\left(\mathrm{~d} \theta^{2}+\sin ^{2} \theta \mathrm{d} \phi^{2}\right) .
\end{aligned}
$$

Now we find the expressions of the density, pressure and electric field:

$\rho=\frac{1}{16 \pi}\left[f_{0}+\left(\frac{1}{r^{2}}-2 T_{0}\right) f_{1}\right]$,

$p=\frac{1}{16 \pi}\left[\left(2 T_{0}-\frac{1}{r^{2}}\right) f_{1}-f_{0}\right]$,

and

$E(r)=\frac{\sqrt{f_{1}}}{\sqrt{2} r}$

So the charge density for the electric field can be expressed as

$\sigma=\sigma_{0} r^{m}\left(\frac{k T_{0} r^{3}+C}{6 r}\right)^{\frac{1}{2}}$

Also the gravitational mass of the interior region can be found:

$$
\begin{aligned}
M(D) & =\int_{0}^{r_{1}=D} 4 \pi r^{2}\left(\rho+\frac{E^{2}}{8 \pi}\right) \mathrm{d} r \\
& =\frac{1}{12}\left(f_{0}-2 f_{1} T_{0}\right) D^{3}+\frac{1}{2} f_{1} D .
\end{aligned}
$$

The gravastar is a singularity-free object, but we observe that a central singularity always occurs at $r=0$. So a gravastar cannot form in the case of $T^{\prime}=0$.

Case II: $f_{T T}=0$.

For $f_{T T}=0$, there are four equations and five unknown functions $a, b, \rho, p, E$. So one function is free. Here we can assume $E$ is a free function of $r$. From Eq. (19), we observe that the expression of $E$ is described by two functions $\sigma$ and $b$. Since $b$ can be determined from the other equations, $\sigma$ is a free function of $r$. In Eq. (19), the functions $\sigma$ and $b$ arise together, so for simplicity of calculation let us assume $\sigma \mathrm{e}^{\frac{b}{2}}=\sigma_{0} r^{m}$ [9] where $\sigma_{0}$ and $m$ are constants, so from Eq. (18) we have

$$
E(r)=E_{0} r^{m+1}
$$

where $E_{0}=\frac{4 \pi \sigma_{0}}{m+3}$. Using Eq. (17), we obtain

$p=-\rho=k_{1} r^{2 m+2}+k_{2}$

where $k_{1}=\frac{(m+3) E_{0}^{2}}{8 \pi(m+1)}$ and $k_{2}$ is constant. From Eq. (24), we obtain

$$
\begin{aligned}
\mathrm{e}^{a}= & k \mathrm{e}^{-b}=k\left[1-\frac{k_{3}}{2 \alpha r}+\frac{\left(\beta+16 \pi k_{2}\right)}{6 \alpha} r^{2}\right. \\
& \left.+\frac{\left(8 \pi k_{1}-E_{0}^{2}\right)}{\alpha(2 m+5)} r^{2 m+4}\right]
\end{aligned}
$$

where $k_{3}$ is an integration constant. We see that, for $k_{3} \neq 0$, the central singularity occurs at $r=0$. Since the gravastar is a singularity-free object, the metric will be non-singular at the center $r=0$. Hence we can choose $k_{3}=0$. So the metric becomes (choose $k=1$ )

$$
\begin{aligned}
\mathrm{d} s^{2}= & -\left[1+\frac{\left(\beta+16 \pi k_{2}\right)}{6 \alpha} r^{2}+\frac{\left(8 \pi k_{1}-E_{0}^{2}\right)}{\alpha(2 m+5)} r^{2 m+4}\right] \mathrm{d} t^{2} \\
& +\left[1+\frac{\left(\beta+16 \pi k_{2}\right)}{6 \alpha} r^{2}+\frac{\left(8 \pi k_{1}-E_{0}^{2}\right)}{\alpha(2 m+5)} r^{2 m+4}\right]^{-1} \mathrm{~d} r^{2} \\
& +r^{2}\left(\mathrm{~d} \theta^{2}+\sin ^{2} \theta \mathrm{d} \phi^{2}\right) .
\end{aligned}
$$

The charge density for the electric field will be

$\sigma=\sigma_{0} r^{m}\left[1+\frac{\left(\beta+16 \pi k_{2}\right)}{6 \alpha} r^{2}+\frac{\left(8 \pi k_{1}-E_{0}^{2}\right)}{\alpha(2 m+5)} r^{2 m+4}\right]^{\frac{1}{2}}$

Also the gravitational mass of the interior region of the gravastar can be found as

$$
\begin{aligned}
M(D) & =\int_{0}^{r_{1}=D} 4 \pi r^{2}\left(\rho+\frac{E^{2}}{8 \pi}\right) \mathrm{d} r \\
& =\frac{\left(E_{0}^{2}-8 \pi k_{1}\right)}{2(2 m+5)} r^{2 m+5}-\frac{4 \pi k_{2}}{3} r^{3} .
\end{aligned}
$$

Thus a gravastar forms in the case of $f_{T T}=0$. In the next subsections, we shall consider only the case $f_{T T}=0$.

\subsection{Shell region}

In this region at $R_{2}\left(D=r_{1}<r<r_{2}=D+\epsilon\right)$, we assume that the thin shell contains a stiff perfect fluid which obeys the $\operatorname{EoS} p=\rho$. For this non-vacuum region, it is very difficult to obtain the general solutions from the field equations. When two regions join together at a place, the intermediate region must be thin shell. So we shall try to find the analytical solution within the thin shell with limit $0<\mathrm{e}^{-b} \equiv h \ll 1$. This thin shell structure suggests that, as $r$ approaches zero, the corresponding radial parameter generally becomes $\ll 1$. 
For $f_{T T}=0$ and in the above approximation (we set $h$ to zero to the leading order), the field equations (24-26) reduce to the following forms [9]:

$-\frac{\alpha h^{\prime}}{r}+\frac{2 \alpha}{r^{2}}+\beta=2 E^{2}$

and

$\alpha h^{\prime}\left(\frac{a^{\prime}}{4}+\frac{1}{2 r}\right)+\frac{\alpha}{r^{2}}=2 E^{2}$.

We see that there are two equations but three unknowns, $a, h$ and $E$. Similar to Eq. (36), let us assume the solution of $E$ in the form $E(r)=E_{0} r^{m+1}$. Solving Eqs. (42) and (43), we obtain

$\mathrm{e}^{-b(r)} \equiv h(r)=h_{2}+2 r+\frac{\beta r^{2}}{2 \alpha}-\frac{E_{0}^{2}}{\alpha(m+2)} r^{2 m+4}$

and

$\mathrm{e}^{a(r)}=\frac{h_{3}}{r^{2}} \operatorname{Exp}\left[\int \frac{8 E_{0}^{2} r^{2 m+4}-4 \alpha}{2 \alpha r+\beta r^{3}-2 E_{0}^{2} r^{2 m+5}} \mathrm{~d} r\right]$

where $h_{2}$ and $h_{3}$ are integration constants and the radius $r$ corresponds to the shell structure in the region $R_{2}$. In this shell region, the range of $r$ is $D<r<D+\epsilon$. Under this assumption $(h \ll 1), \epsilon \ll 1$, so we must have $h_{2} \ll 1$. From Eq. (25) we obtain

$8 \pi p=8 \pi \rho=E_{0}^{2} r^{2 m+2}-\frac{\alpha}{r^{2}}-\frac{\beta}{2}$.

Also the charge density for electric field is given by

$\sigma=\sigma_{0} r^{m}\left[h_{2}+2 r+\frac{\beta r^{2}}{2 \alpha}-\frac{E_{0}^{2}}{\alpha(m+2)} r^{2 m+4}\right]^{\frac{1}{2}}$.

\subsection{Exterior region}

For the exterior region $R_{3}\left(r>r_{2}=D+\epsilon\right)$, the vacuum EoS is given by $p=\rho=0$. In this region, from Eq. (18), we obtain

$E(r)=\frac{Q}{r^{2}}$

where $Q$ is constant electric charge. From Eq. (17), we obtain

$\mathrm{e}^{b(r)}=k \mathrm{e}^{-a(r)}$

where $k$ is a constant $>0$.
For $f_{T T}=0$, we obtain the solutions

$\mathrm{e}^{a}=k \mathrm{e}^{-b}=k\left(1-\frac{2 M}{r}+\frac{\beta}{6 \alpha} r^{2}+\frac{Q^{2}}{\alpha r^{2}}\right)$

where $M$ is the mass of the gravastar. Also the charge density for the electric field may be written as

$\sigma=\sigma_{0} r^{m}\left(1-\frac{2 M}{r}+\frac{\beta}{6 \alpha} r^{2}+\frac{Q^{2}}{\alpha r^{2}}\right)^{\frac{1}{2}}$

So in the exterior region the metric becomes (choose $k=$ 1)

$$
\begin{aligned}
\mathrm{d} s^{2}= & -\left(1-\frac{2 M}{r}+\frac{\beta}{6 \alpha} r^{2}+\frac{Q^{2}}{\alpha r^{2}}\right) \mathrm{d} t^{2} \\
& +\left(1-\frac{2 M}{r}+\frac{\beta}{6 \alpha} r^{2}+\frac{Q^{2}}{\alpha r^{2}}\right)^{-1} \mathrm{~d} r^{2} \\
& +r^{2}\left(\mathrm{~d} \theta^{2}+\sin ^{2} \theta \mathrm{d} \phi^{2}\right) .
\end{aligned}
$$

For $\alpha=1$ and $\beta=0$, we get the usual Reissner-Nordstrom spacetime metric and, for $\alpha=1, \beta=0$ and $Q=0$, we get back the static Schwarzschild metric.

\section{Physical features}

Now we shall discuss the physical features of the parameters of the gravastar shell region, like the proper length of the shell, the energy and the entropy within the shell.

\subsection{Proper length}

Since the stiff perfect fluid propagates between two boundaries of the shell region of the gravastar, the inner boundary of the shell is located at the surface $r=D$ and the outer boundary of the shell is located at the surface $r=D+\epsilon$, where the proper thickness of the shell is assumed to be very small, i.e., $\epsilon \ll 1$. So, the proper thickness of the shell is determined by [9]

$\ell=\int_{D}^{D+\epsilon} \sqrt{\mathrm{e}^{b(r)}} \mathrm{d} r$

Since in the shell region, the expressions of $\mathrm{e}^{b(r)}$ are lengthy, we cannot find the analytical form of the above integral. So let us assume $\sqrt{\mathrm{e}^{b(r)}}=\frac{\mathrm{d} g(r)}{\mathrm{d} r}$, so from the above integral we get

$$
\begin{aligned}
\ell & =\int_{D}^{D+\epsilon} \frac{\mathrm{d} g(r)}{\mathrm{d} r} \mathrm{~d} r=g(D+\epsilon)-\left.g(D) \approx \epsilon \frac{\mathrm{d} g(r)}{\mathrm{d} r}\right|_{D} \\
& =\epsilon \sqrt{\mathrm{e}^{b(D)}}
\end{aligned}
$$


where we have taken only the first order term of $\epsilon$ (since $\epsilon \ll 1$, we have $O\left(\epsilon^{2}\right) \approx 0$ ). For $f_{T T}=0$, the proper length will be (from Eq. (44))

$\ell=\epsilon\left[h_{2}-2 D-\frac{\beta D}{2}+\frac{E_{0}^{2}}{\alpha(m+2)} D^{2 m+4}\right]^{-\frac{1}{2}}$,

which implies that the proper length of the shell is proportional to the thickness $(\epsilon)$ of the shell.

\subsection{Energy}

The energy within the shell region of the gravastar is [9]

$\mathcal{E}=\int_{D}^{D+\epsilon} 4 \pi r^{2}\left[\rho+\frac{E^{2}}{8 \pi}\right] \mathrm{d} r$

For $f_{T T}=0$, we can obtain the energy as

$$
\begin{aligned}
\mathcal{E}= & \int_{D}^{D+\epsilon}\left[E_{0}^{2} r^{2 m+4}-\frac{\alpha}{2}-\frac{\beta r^{2}}{4}\right] \mathrm{d} r \\
= & \frac{E_{0}^{2}}{2 m+4}\left[(D+\epsilon)^{2 m+4}-D^{2 m+4}\right] \\
& -\frac{\epsilon \alpha}{2}-\frac{\beta}{12}\left[(D+\epsilon)^{3}-D^{3}\right] \\
\approx & \epsilon E_{0}^{2} D^{2 m+4}-\frac{\epsilon \alpha}{2}-\frac{\epsilon \beta D^{2}}{4} .
\end{aligned}
$$

For this approximation, we see that the energy content in the shell is proportional to the thickness $(\epsilon)$ of the shell.

\subsection{Entropy}

Mazur and Mottola $[1,2]$ have shown that the entropy density is zero in the interior region $R_{1}$ of the gravastar. However, the entropy within the shell can be defined by [9]

$S=\int_{D}^{D+\epsilon} 4 \pi r^{2} s(r) \sqrt{\mathrm{e}^{b(r)}} \mathrm{d} r$

where $s(r)$ is the entropy density for the local temperature $T(r)$ and which can be written as

$s(r)=\frac{\gamma^{2} k_{B}^{2} T(r)}{4 \pi \hbar}=\frac{\gamma k_{B}}{\hbar} \sqrt{\frac{p(r)}{2 \pi}}$

where $\gamma$ is a dimensionless constant. So the entropy can be written as

$S=\frac{\gamma k_{B}}{\hbar} \int_{D}^{D+\epsilon} r^{2} \sqrt{8 \pi p(r) \mathrm{e}^{b(r)}} \mathrm{d} r$
For the approximation $(\epsilon \ll 1)$, we can obtain

$S \approx \frac{\epsilon \gamma k_{B}}{\hbar} D^{2} \sqrt{8 \pi p(D) \mathrm{e}^{b(D)}}$

For $f_{T T}=0$, we get the entropy as

$$
\begin{aligned}
S \approx & \frac{\epsilon \gamma k_{B}}{\hbar}\left[E_{0}^{2} D^{2 m+3}-\frac{\alpha}{D}-\frac{\beta D}{2}\right]^{\frac{1}{2}} \\
& \times\left[h_{2}-2 D-\frac{\beta D}{2}+\frac{E_{0}^{2}}{\alpha(m+2)} D^{2 m+4}\right]^{-\frac{1}{2}} .
\end{aligned}
$$

This means that the entropy in the shell is proportional to the thickness $(\epsilon)$ of the shell.

\section{Junction conditions between interior and exterior regions}

Since a gravastar consists of three regions: the interior region, the shell region and the exterior region, it is necessary to match the surfaces of the interior and exterior regions according to the Darmois-Israel formalism [51-53]. At $r=D$, the junction surface is denoted by $\Sigma$. We consider the metric on the junction surface in the form

$d s^{2}=-f(r) \mathrm{d} t^{2}+\frac{\mathrm{d} r^{2}}{f(r)}+r^{2}\left(\mathrm{~d} \theta^{2}+\sin ^{2} \theta \mathrm{d} \phi^{2}\right)$

where the metric coefficients are continuous at $\Sigma$, though their derivatives may not be continuous at $\Sigma$. With the help of the Darmois-Israel formalism, we want to find the expression for the stress-energy surface $S_{i j}$ from the Lanczos equation [54],

$S_{j}^{i}=-\frac{1}{8 \pi}\left(\eta_{j}^{i}-\delta_{j}^{i} \eta_{k}^{k}\right)$

where $\eta_{i j}=K_{i j}^{+}-K_{i j}^{-}$. Here $K_{i j}$ is the extrinsic curvature. So $\eta_{i j}$ gives the discontinuous surfaces in the extrinsic curvatures (second fundamental form). Here the signs " + " and " - " correspond to the interior and the exterior regions of the gravastar, respectively. The extrinsic curvatures associated with the two surfaces of the shell region can be written as

$K_{i j}^{ \pm}=\left[-n_{v}^{ \pm}\left\{\frac{\partial^{2} x_{v}}{\partial \xi^{i} \partial \xi^{j}}+\Gamma_{\alpha \beta}^{v} \frac{\partial x^{\alpha}}{\partial \xi^{i}} \frac{\partial x^{\beta}}{\partial \xi^{j}}\right\}\right]_{\Sigma}$

where $\xi^{i}$ are the intrinsic coordinates on the shell, and $n_{v}^{ \pm}$ are the unit normals to the surface $\Sigma$, defined by $n_{v} n^{v}=-1$. For the above metric, we obtain

$n_{v}^{ \pm}= \pm\left[g^{\alpha \beta} \frac{\partial f}{\partial x^{\alpha}} \frac{\partial f}{\partial x^{\beta}}\right]^{-\frac{1}{2}} \frac{\partial f}{\partial x^{\nu}}$. 
Using the Lanczos equation, we can obtain the stress-energy surface tensor as $S_{j}^{i}=\operatorname{diag}(-\varrho, \wp, \wp, \wp)$ where $\varrho$ is the surface energy density and $\wp$ is the surface pressure given by [10]

$\varrho=-\frac{1}{4 \pi D}[\sqrt{f}]_{-}^{+}$

and

$\wp=\frac{1}{8 \pi D}[\sqrt{f}]_{-}^{+}+\frac{1}{16 \pi}\left[\frac{f^{\prime}}{\sqrt{f}}\right]_{-}^{+}$.

For $f_{T T}=0$, we obtain

$$
\begin{aligned}
\varrho= & -\frac{1}{4 \pi D}\left[\sqrt{1-\frac{2 M}{D}+\frac{\beta D^{2}}{6 \alpha}+\frac{Q^{2}}{\alpha D^{2}}}\right. \\
& -\sqrt{1+\frac{\left(\beta+16 \pi k_{2}\right) D^{2}}{6 \alpha}+\frac{\left(8 \pi k_{1}-E_{0}^{2}\right)}{\alpha(2 m+5)} D^{2 m+4}}
\end{aligned}
$$

and

$$
\begin{aligned}
\wp= & \frac{1}{8 \pi D}\left[\sqrt{1-\frac{2 M}{D}+\frac{\beta D^{2}}{6 \alpha}+\frac{Q^{2}}{\alpha D^{2}}}\right. \\
& \left.-\sqrt{1+\frac{\left(\beta+16 \pi k_{2}\right) D^{2}}{6 \alpha}+\frac{\left(8 \pi k_{1}-E_{0}^{2}\right)}{\alpha(2 m+5)} D^{2 m+4}}\right] \\
+ & \frac{1}{16 \pi}\left[\frac{\frac{2 M}{D^{2}}+\frac{\beta D}{3 \alpha}-\frac{2 Q^{2}}{\alpha D^{3}}}{\sqrt{1-\frac{2 M}{D}+\frac{\beta D^{2}}{6 \alpha}+\frac{Q^{2}}{\alpha D^{2}}}}\right. \\
& \left.-\frac{\left(\beta+16 \pi k_{2}\right) D}{3 \alpha+\frac{(2 m+4)\left(8 \pi k_{1}-E_{0}^{2}\right)}{\alpha(2 m+5)} D^{2 m+3}}\right] .
\end{aligned}
$$

\subsection{Equation of state}

The equation of state parameter $w(D)$ can be written as

$w(D)=\frac{\wp}{\varrho}$

For $f_{T T}=0$, the equation of state parameter can be written in the following form:

$w(D)=-\frac{1}{2}-\frac{1}{4}\left[\frac{\frac{2 M}{D^{2}}+\frac{\beta D}{3 \alpha}-\frac{2 Q^{2}}{\alpha D^{3}}}{\sqrt{1-\frac{2 M}{D}+\frac{\beta D^{2}}{6 \alpha}+\frac{Q^{2}}{\alpha D^{2}}}}\right.$

$$
\begin{aligned}
& -\frac{\frac{\left(\beta+16 \pi k_{2}\right) D}{3 \alpha}+\frac{(2 m+4)\left(8 \pi k_{1}-E_{0}^{2}\right)}{\alpha(2 m+5)} D^{2 m+3}}{\left.\sqrt{1+\frac{\left(\beta+16 \pi k_{2}\right) D^{2}}{6 \alpha}+\frac{\left(8 \pi k_{1}-E_{0}^{2}\right)}{\alpha(2 m+5)} D^{2 m+4}}\right]} \\
& \times\left[\sqrt{1-\frac{2 M}{D}+\frac{\beta D^{2}}{6 \alpha}+\frac{Q^{2}}{\alpha D^{2}}}\right. \\
& \left.-\sqrt{1+\frac{\left(\beta+16 \pi k_{2}\right) D^{2}}{6 \alpha}+\frac{\left(8 \pi k_{1}-E_{0}^{2}\right)}{\alpha(2 m+5)} D^{2 m+4}}\right]^{-1} .
\end{aligned}
$$

\subsection{Mass}

The mass $\mathcal{M}$ of the thin shell can be obtained from the following formula:

$\mathcal{M}=4 \pi D^{2} \varrho$

For $f_{T T}=0$, the mass of the thin shell can be expressed as

$$
\begin{aligned}
\mathcal{M}= & -D\left[\sqrt{1-\frac{2 M}{D}+\frac{\beta D^{2}}{6 \alpha}+\frac{Q^{2}}{\alpha D^{2}}}\right. \\
& \left.-\sqrt{1+\frac{\left(\beta+16 \pi k_{2}\right) D^{2}}{6 \alpha}+\frac{\left(8 \pi k_{1}-E_{0}^{2}\right)}{\alpha(2 m+5)} D^{2 m+4}}\right] .
\end{aligned}
$$

So the total mass $M$ of the gravastar in terms of the thin shell can be expressed as

$$
\begin{aligned}
M= & \frac{D}{2}+\frac{\beta D^{3}}{12 \alpha}+\frac{Q^{2}}{2 \alpha D} \\
& -\frac{D}{2}\left[\sqrt{1+\frac{\left(\beta+16 \pi k_{2}\right) D^{2}}{6 \alpha}+\frac{\left(8 \pi k_{1}-E_{0}^{2}\right)}{\alpha(2 m+5)} D^{2 m+4}}-\frac{\mathcal{M}}{D}\right]^{2} .
\end{aligned}
$$

We see that the total mass $M$ of the gravastar will be less than $\frac{D}{2}+\frac{\beta D^{3}}{12 \alpha}+\frac{Q^{2}}{2 \alpha D}$.

\section{Discussions}

In the present work, we have discussed the four dimensional spherically symmetric stellar system in the framework of modified $f(T)$ gravity theory with an electromagnetic field. The field equations have been found for two cases, $T^{\prime}=0$ and $f_{T T}=0$. For $T^{\prime}=0, T$ must be constant and all the derivatives of $f(T)$ with respect to $T$ must be constants. Also for $f_{T T}=0$, we have found that $f(T)$ is a linear function of $T$. Next we have discussed the charged gravastar model 
where the equation of state in the three regions of the gravastar satisfies for the interior region $(p=-\rho)$, for the shell region $(p=\rho)$ and for the exterior region $(p=\rho=0)$. In the interior region, we have found the solutions of all physical quantities like density, pressure, electromagnetic field and also the metric coefficients for both cases. For $T^{\prime}=0$, we have found $E(r) \propto \frac{1}{r}$. Since for $T^{\prime}=0$, the central singularity occurs at $r=0$, a gravastar cannot form in this case. For $f_{T T}=0, E(r) \propto r^{m+1}$ and gravastar forms in this case. In the exterior region, we have obtained the exterior solution for the vacuum model. For $f_{T T}=0$, we found that the metric is a generalization of the Reissner-Nordstrom spacetime. In the shell region, we have assumed that the interior and exterior regions join together at a certain place, so the intermediate region must be a thin shell with limit $0<\mathrm{e}^{-b} \equiv h \ll 1$. This thin shell structure suggests that, as $r$ approaches zero, the corresponding radial parameter generally becomes $\ll 1$. In this approximation, we have found the solutions for $f_{T T}=0$. The electromagnetic field gets the form $E \propto r^{m+1}$. The proper length of the thin shell, the entropy and the energy content inside the thin shell have been found and they are directly proportional to the proper thickness of the shell $\epsilon$ under the approximation $(\epsilon \ll 1)$. According to the Darmois-Israel formalism, we have studied the matching between the surfaces of the interior and exterior regions of the gravastar. The energy density and pressure on the surface have been obtained. Also the equation of state parameter $w(D)$ has been found. Moreover, the mass $\mathcal{M}$ of the thin shell has been obtained and the total mass of the gravastar has been expressed in terms of the thin shell mass.

Data Availability Statement This manuscript has no associated data or the data will not be deposited. [Authors' comment: This article describes only theoretical, so data sharing is not applicable in the current study.]

Open Access This article is distributed under the terms of the Creative Commons Attribution 4.0 International License (http://creativecomm ons.org/licenses/by/4.0/), which permits unrestricted use, distribution, and reproduction in any medium, provided you give appropriate credit to the original author(s) and the source, provide a link to the Creative Commons license, and indicate if changes were made.

Funded by SCOAP ${ }^{3}$.

\section{References}

1. P. Mazur, E. Mottola, Report number: LA-UR-01- 5067 (arXiv:gr-qc/0109035)

2. P. Mazur, E. Mottola, Proc. Natl. Acad. Sci. USA. 101, 9545 (2004)

3. M. Visser, D.L. Wiltshire, Class. Quantum Gravity 21, 1135 (2004)

4. A. DeBenedictis, D. Horvat, S. Ilijic, S. Kloster, K.S. Viswanathan, Class. Quantum Gravity 23, 2303 (2006)

5. C. Cattoen, T. Faber, M. Visser, Class. Quantum Gravity 22, 4189 (2005)

6. N. Bilic, G.B. Tupper, R.D. Viollier, JCAP 0602, 013 (2006)
7. F. Rahaman, S. Chakraborty, S. Ray, A.A. Usmani, S. Islam, Int. J. Theor. Phys. 54, 50 (2015)

8. P. Bhar, Astrophys. Space Sci. 354, 2109 (2014)

9. S. Ghosh, F. Rahaman, B.K. Guha, S. Ray, Phys. Lett. B 767, 380 (2017)

10. S. Ghosh, S. Ray, F. Rahaman, B.K. Guha, Ann. Phys. 394, 230 (2018)

11. A.A. Usmani, F. Rahaman, S.A. Rakib, S. Ray, K.K. Nandi, P.K.F. Kuhfittig, Z. Hasan, Phys. Lett. B 701, 388 (2011)

12. A. Banerjee, F. Rahaman, S. Islam, M. Govender, Eur. Phys. J. C 76, 34 (2016)

13. D. Horvat, S. Ilijic, A. Marunovic, Class. Quantum Gravity 26(025003), 025003 (2009)

14. R. Chan, M.F.A. da Silva, JCAP 1007, 029 (2010)

15. F. Rahaman, A.A. Usmani, S. Ray, S. Islam, Phys. Lett. B 717, 1 (2012)

16. C.F.C. Brandt, R. Chan, M.F.A. da Silva, P. Rocha, J. Mod. Phys. 6, 879 (2013)

17. A. Ovgun, A. Banerjee, K. Jusufi, Eur. Phys. J. C 77, 566 (2017)

18. B.M.N. Carter, Class. Quantum Gravity 22, 4551 (2005)

19. P. Rocha, A.Y. Miguelote, R. Chan, M.F. da Silva, N.O. Santos, A. Wang, JCAP 0806, 025 (2008)

20. P. Rocha, R. Chan, M.F.A. da Silva, A. Wang, JCAP 0811, 010 (2008)

21. R. Chan, M.F.A. da Silva, P. Rocha, A. Wang, JCAP 0903, 010 (2009)

22. A. Banerjee, J.R. Villanueva, P. Channuie, K. Jusuf, Chin. Phys. C 42, 115101 (2018)

23. S. Nojiri, S.D. Odintsov, Int. J. Geom. Methods Mod. Phys. 4, 115 (2007)

24. S. Capozziello, M. Francaviglia, Gen. Relativ. Gravit. 40, 357 (2008)

25. T.P. Sotiriou, V. Faraoni, Rev. Mod. Phys. 82, 451 (2010)

26. A. De Felice, S. Tsujikawa, Living Rev. Relativ. 13, 3 (2010)

27. A. Einstein, Sitz. Preuss. Akad. Wiss. 217, (1928)

28. A. Einstein, Sitz. Preuss. Akad. Wiss. 224, (1928)

29. A. Einstein, Math. Ann. 102, 685 (1930)

30. A. Unzicker, T. Case, arXiv:physics/0503046v1

31. V.C. De Andrade, L.C.T. Guillen, J.G. Pereira, arXiv:gr-qc/0011087

32. G.R. Bengochea, R. Ferraro, Phys. Rev. D 79, 124019 (2009)

33. J.B. Dent, S. Dutta, E.N. Saridakis, JCAP 1101, 009 (2011)

34. T. Wang, Phys. Rev. D 84, 024042 (2011)

35. C. Deliduman, B. Yapiskan, arXiv:1103.2225 [gr-qc]

36. C.G. Boehmer, A. Mussa, N. Tamanini, Class. Quantum Gravity 28, 245020 (2011)

37. G. Abbas, S. Qaisar, M.A. Meraj, Astrophys. Space Sci. 357, 156 (2015)

38. P. Saha, U. Debnath, Adv. High Energy Phys. 2018, 3901790 (2018)

39. G. Abbas, S. Qaisar, W. Javed, M.A. Meraj, Iran. J. Sci. Technol. A 42, 1659 (2016)

40. M.G. Ganiou, C. Anamon, M.J.S. Houndjo, J. Tossa, Eur. Phys. J. Plus 132, 250 (2017)

41. A.V. Kpadonou, M.J.S. Houndj, M.E. Rodrigues, Astrophys. Space Sci. 361, 244 (2016)

42. G. Abbas, S. Nazeer, M.A. Meraj, Astrophys. Space Sci. 354, 449 (2014)

43. G. Abbas, A. Kanwal, M. Zubair, Astrophys. Space Sci. 357, 109 (2015)

44. G. Abbas et al., Astrophys. Space Sci. 357, 158 (2015)

45. G. Abbas, M. Zubair, G. Mustafa, Astrophys. Space Sci. 358, 26 (2015)

46. K.D. Krori, J. Barua, J. Phys. A Math. Gen. 8, 508 (1975)

47. M.F. Shamir, M. Ahmad, Phys. Rev. D 97, 104031 (2018)

48. A. Das, S. Ghosh, B.K. Guha, S. Das, F. Rahaman, S. Ray, Phys. Rev. D 95, 124011 (2017) 
49. T.P. Sotiriou, V. Faraoni, Rev. Mod. Phys. 82, 451 (2010)

50. K. Bamba, S. Capozziello, S. Nojiri, S.D. Odintsov, Astrophys. Space Sci. 345, 155 (2012)

51. G. Darmois, Memorial des sciences mathematiques XXV, Fasticule XXV, (Gauthier-Villars, Paris, France, 1927), chap. V
52. W. Israel, Nuovo Cimemto 44, 1 (1966)

53. W. Israel, Nuovo Cimemto 48, 463(E) (1967)

54. K. Lanczos, Ann. Phys. 379, 518 (1924) 